\title{
Human genes in TB infection: their role in immune response
}

\author{
D. Lykouras ${ }^{1}$, F. Sampsonas 1 , A. Kaparianos 1 , K. Karkoulias 1 , \\ G. Tsoukalas'2, K. Spiropoulos ${ }^{1}$
}

\begin{abstract}
Human genes in TB infection: their role in immune response. D. Lykouras, F. Sampsonas, A. Kaparianos, K. Karkoulias, G. Tsoukalas, K. Spiropoulos.

Tuberculosis (TB) caused by the human pathogen Mycobacterium tuberculosis, is the leading cause of morbidity and mortality caused by infectious agents worldwide. Recently, there has been an ongoing concern about the clarification of the role of specific human genes and their polymorphisms involved in TB infection. In the vast majority of individuals, innate immune pathways and $\mathrm{T}$ helper 1 (Th1) cell mediated immunity are activated resulting in the lysis of the bacterium.

Firstly, PTPN22 R620W polymorphism is involved in the response to cases of infection. The Arg753GIn polymorphism in TLR-2 leads to a weaker response against the
\end{abstract}

M. tuberculosis. The gene of the vitamin D receptor (VDR) has a few polymorphisms (BsmI, ApaI, Taq1, FokI) whose mixed genotypes alter the immune response. Solute carrier family 11 member (SLC11A1) is a proton/divalent cation antiporter that is more familiar by its former name NRAMP1 (natural resistance associated macrophage protein 1) and can affect $M$. tuberculosis growth.

Polymorphisms of cytokines such as IL-10, IL-6, IFN-g, TNF-a, TGF-b1 can affect the immune response in various ways. Finally, a major role is played by $M$. tuberculosis antigens and the Ras-associated small GTP-ase 33A.

As far as we know this is the first review that collates all these polymorphisms in order to give a comprehensive image of the field, which is currently evolving. Monaldi Arch Chest Dis 2008; 69: 1, 24-31.

Keywords: TB, Genes, Polymorphism, Vitamin D, Cytokines.

1 University of Patras, University Hospital of Patras, Department of Internal Medicine, Division of Pneumology, Rio, Patras,

2 Chest Hospital Sotiria, Athens, Greece.

Correspondence: Kostas Spiropoulos, Professor of Pneumology, University Hospital of Patras, Rio, Patras 26500, Greece; e-mail: k-spiropoulos@hotmail.com

\section{Introduction}

Tuberculosis (TB) caused by the human pathogen Mycobacterium tuberculosis, is the leading cause of morbidity and mortality caused by infectious agents worldwide.

Eight million new cases of tuberculosis (TB), along with 2-3 million TB-related deaths, are recorded each year, and it is estimated that onethird of the world's population is presently infected with Mycobacterium tuberculosis [1]. Fortunately, not all individuals infected with $\mathrm{M}$. tuberculosis will develop overt disease, and 90\%-95\% of infected individuals will stay healthy throughout their lives. In contrast to individuals with active $\mathrm{TB}$, the vast majority of individuals with latent TB can therefore be considered to be protected by an appropriate, naturally acquired immune response to $\mathrm{M}$. tuberculosis antigens.

Tuberculosis is primarily acquired through inhalation of airborne droplets containing Mycobacterium tuberculosis (Mtb) (fig. 1). The bacteria travel to distal regions of the lung and are engulfed by lung dendritic cells (DCs) and macrophages. In the vast majority of individuals, an orchestrated series of innate immune pathways and T-helper 1 (Th1)-dominant adaptive immune pathways are activated following phagocytosis of the bacteria, to culminate in a granuloma at the initial focus of infection [2].

Many studies, notably those on monozygotic and dizygotic twins, indicate that inherited genetic factors play a key role in determining susceptibility and resistance to overt TB following infection [3]. The immune interactions between the host and the highly complex molecular structure of M. tuberculosis are multifactorial.

At the site of multiplication of bacilli, neutrophils are the first cells to be recruited, followed by NK cells, $\gamma / \delta$ T cells, and $\alpha / \beta$ T cells [4]. Studies in humans have demonstrated that neutrophils provide agents such as defensins, which are lacking in macrophage-mediated killing [5]. NK cells are also the effector cells of innate immunity. These cells may directly cause the lysis of pathogens or that of infected monocytes. In vitro co-culture with live $\mathrm{M}$. tuberculosis results in the expansion of NK cells, implying that they may be important responders to M. tuberculosis infection in vivo. During early infection, NK cells are capable of activating phagocytic cells at the site of infection [6].

From the standpoint of the host, resistance to infection is dependent on the ability of macro- 
phages to phagocytose and destroy the bacilli; and this, in turn, is affected by class II HLA-determined antigen presentation, the activation of macrophages by T cell-derived cytokines and vitamin D [7], and granuloma formation. Several of the genes involved in these processes have been identified, notably the HLA-DR and HLA-DQB1 loci, which determine which mycobacterial antigens are presented to helper T cells.

But, the diagnosis of $\mathrm{M}$. tuberculosis infection is more difficult to establish than with almost every other common bacterial infection [8] and it seems that the study of genes involved in the procedures of the infection will give the possibility both to diagnose and try curing tuberculosis. Supposing we know the genetic profile of the patient we will be able to make prompt and accurate prognosis. Moreover screening of the population will lead to the restriction of epidemics of certain strains.

\section{Genes and their polymorphisms associated to Tuberculosis}

\section{HLA haplotypes gene and the association with TB}

At the beginning of the host immune response the HLA-complex system is involved in the presentation of the antigen. Thus, its association is undoubted and table 1 illustrates some of the studied HLA haplotypes appearing in TB infected patients worldwide, as it would take too long to discuss this subject thoroughly.

Nevertheless, MHC genes are not sufficient in conferring susceptibility or resistance to disease. However, non-HLA genes have also been implicated in TB susceptibility.

Table 1. - HLA haplotypes associated with pulmonary TB

\begin{tabular}{ll}
\hline $\begin{array}{l}\text { HLA haplotype associated } \\
\text { with pulmonary TB }\end{array}$ & Population \\
\hline HLA-DRB $1 * 1501$ & Asian Indians [9] \\
HLA-DQB $1 * 0502$ & Asian Indians \\
HLA-DQB $1 * 0503$ & Vietnam [10] \\
HLA-DQB $1 * 05$ & Poland [11] \\
HLA-DQB $1 * 02$ & Poland \\
\hline
\end{tabular}

\section{PTPN22 R620W Polymorphism in TB}

The PTPN22 gene product (i.e., Lyp) is an important downregulator of T-cell activation and other cell subsets such as NK cells and neutrophils, the action is performed through physical interaction with Csk and probably through its interaction with the adaptor molecule Grb2 [12, 13].

Remarkably, the association of the PTPN22 polymorphism with disease (table 2) in the population selected by Gomez et al. [14] is opposite to that of TB and autoimmunity, suggesting positive selection. Natural selection for resistance to a pathogen can increase the frequency of alleles that are otherwise deleterious. Thus, infectious diseases such as TB may exert immunological protection against autoimmunity (by homeostatic competition, bystander suppression, or toll-like receptor stimulation), favouring at the same time, a selective genetic pressure that would increase the risk of autoimmune diseases.

\section{Polymorphism in TLR-2}

TLRs comprise a family of mammalian cellsurface proteins that stimulate pro-inflammatory cytokine gene transcription in response to various microbial ligands. TLRs mediate cellular responses to microorganism, but are not required for phagocytosis. Members of the mammalian TLR family have been implicated in the activation of macrophages by a variety of chemically diverse

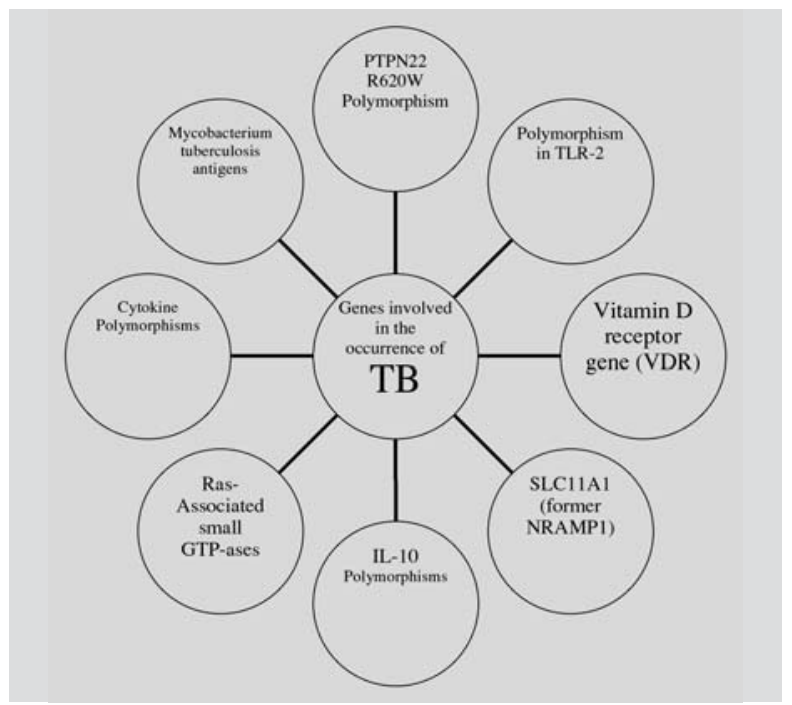

Fig. 1. - Genes and their polymorphisms associated to Tuberculosis.

Table 2. - PTPN22 R620W polymorphism and the association of its carriers with TB

\begin{tabular}{lll}
\hline & T allele carriers & C allele carriers \\
\hline Tuberculin skin test positive (TST+) & Protected against disease & Prone to development of overt TB \\
\hline Tuberculin skin test negative (TST-) & $\begin{array}{l}\text { Comlementary mechanisms active, } \\
\text { or never contacted the M. tuberculosis }\end{array}$ & \\
\hline
\end{tabular}


bacterial products $[15,16]$. TLR2 is involved in the recognition of various lipoproteins of microorganisms such as Borrelia, Mycoplasma and Treponema, and certainly $M$. tuberculosis [17]. Blocking this receptor abolishes the ability of macrophages to sense and respond to mycobacterium. Macrophages sense the presence of mycobacteria through activation of TLR2 (firstline defence), triggering activation of further immune response (TNF-a, interleukin-1 production, antigen processing, activation of T-lymphocytes, interferon gamma production, etc.) in later events. In vitro studies have shown that TLR2 activation directly leads to intracellular killing of M. tuberculosis by alveolar macrophages [18].

Lorenz et al. [19] reported a novel polymorphism in the TLR2 gene (arginine to glutamine substitution at residue 753 (Arg753Gln)) that leads to a decreased response of macrophages to bacterial peptides, resulting in an attenuated immune response in the host.

Ogus et al. [20] have studied the Arg753Gln Polymorphism in TLR-2 (table 3).

This TLR2 gene polymorphism may be one of the factors influencing disease susceptibility, although other factors or defects in different steps of the immune response (even other polymorphisms of TLR2 or other TLRs) might also be responsible for an inability to prevent progression of TB infection to disease.

TLR activation of human macrophages upregulates expression of the vitamin $\mathrm{D}$ receptor and the vitamin D-1-hydroxylase genes, leading to induction of the antimicrobial peptide cathelicidin and killing of intracellular Mycobacterium tuberculosis. Moreover, sera from African-American individuals, known to have increased susceptibility to tuberculosis, had low 25-hydroxyvitamin D and were inefficient in supporting cathelicidin messenger RNA induction. Thus, there is a link between TLRs and vitamin D-mediated innate immunity suggesting that differences in ability of human populations to produce vitamin D may contribute to susceptibility to microbial infection [21].

\section{The role of vitamin $D$ receptor $(V D R)$ gene}

Vitamin D receptor (VDR) gene is one of the important candidate genes (non-MHC gene) being studied in tuberculosis. The importance of polymorphic variant genotypes of VDR gene has been emphasised on the susceptibility or resistance to

Table 3. - The Arg753Gln Polymorphism in TLR-2 has genotypes showing certain connection with TB occurance

\begin{tabular}{ll}
\hline Genotype & Risk of TB \\
\hline GG & Healthy \\
GA & 1,60 fold higher risk than in GG carriers \\
AA & 6,04 fold higher risk than in GG carr \\
\hline
\end{tabular}

various infectious diseases including pulmonary tuberculosis (PTB) [22]. Before the discovery of effective antimycobacterial drugs, vitamin D3 was used to treat patients with cutaneous TB with striking effects and vitamin D3 therapy in the form of cod liver oil and exposure to sunlight were used to treat human tuberculosis. 1.25-dihydroxy vitamin D3 is an important immunomodulatory hormone, which activates monocytes and suppresses lymphocyte proliferation, immunoglobulin production, and cytokine synthesis [23]. Moreover, this hormone has been shown to act as both an "upregulating agent" during natural immunity via the enhancement of phagocytosis by monocyte/ macrophage populations and a "downregulator" during acquired immune responses via an inhibitory effect on MHC Class II expression by antigen presenting cells.

\section{Vitamin D receptor and the translocation of the receptor into nucleus}

Vitamin D3 exerts its action through VDR, a nuclear hormone receptor (fig. 2). VDR is present on monocytes and activated $\mathrm{T}$ and $\mathrm{B}$ lymphocytes. VDR is a ligand activated transcription factor, which upon binding with vitamin D3, heterodimerises with Retinoid X Receptor and interacts with Vitamin D response elements, upstream of target genes and alters their transcription [24]. Several polymorphisms have been identified in the VDR gene, most of which are identified by A biallelic variation in restriction enzyme sites, which include the BsmI, ApaI at intron 8 ( $\mathrm{T}$ to $\mathrm{G}$ ), a silent TaqI site in exon 9 ( $\mathrm{T}$ to $\mathrm{C}$ ) and a functional FokI site ( $\mathrm{C}$ to $\mathrm{T})$. Altered VDR mRNA expression has been shown with variant genotypes of the VDR gene [25] (tables 4, 5).

The observed unresponsive nature of the macrophages and lymphocytes to vitamin D3 stimulation in PTB patients, irrespective of the

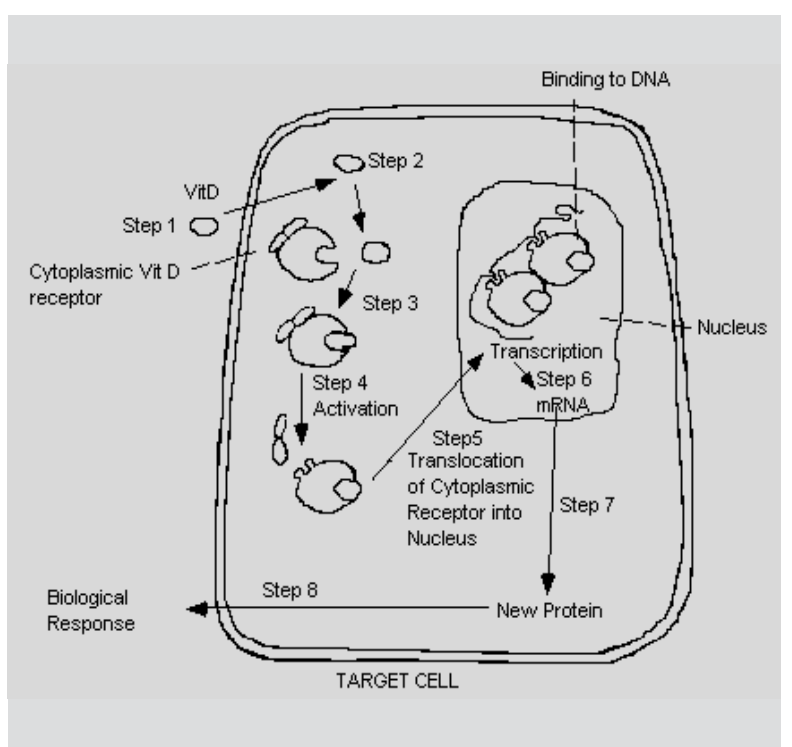

Fig. 2. - Vitamin D receptor and the translocation of the receptor into nucleus. 
Table 4. - VDR gene polymorphisms and their alleles

\begin{tabular}{lllll}
\hline & \multicolumn{4}{c}{ Genes } \\
\cline { 2 - 5 } & BsmI & ApaI & TaqI & FokI \\
\hline Alleles & BB & AA & TT & FF \\
& Bb & Aa & tt & Ff \\
\hline
\end{tabular}

Table 5. - VDR gene polymorphisms and the association of certain alleles and genotypes with TB

\begin{tabular}{ll}
\hline Genotype & VDR expression \\
\hline $\mathrm{BB}$ & Increased \\
$\mathrm{Bb}$ & Normal \\
$\mathrm{AA}$ & Normal \\
$\mathrm{Aa}$ & Normal \\
$\mathrm{TT}$ & Normal \\
$\mathrm{tt}$ & Increased \\
$\mathrm{FF}$ & Increased \\
$\mathrm{Ff}$ & Normal \\
BBAAtt & Increased \\
BAt & Increased \\
\hline
\end{tabular}

variant genotypes of VDR gene, may be due to increased level of endogenous secretion of vitamin D3 by activated alveolar macrophages and other cells which is suggested to be associated with hypercalcemia in tuberculosis [26]. High level of endogenous vitamin D3 during active stage of tuberculosis may downregulate the expression of VDRs and make the cells refractory to external administration of vitamin D3 under in vitro condition.

\section{SLC11A1 (NRAMP1) gene and the association with TB}

Solute carrier family 11 member 1 protein (SLC11A1), formerly called NRAMP1 (for Natural Resistance Associated Macrophage Protein 1), is a protein with highly conserved amino acid sequence across species [27]. The SLC11A1 gene, located on $2 \mathrm{q} 35$, is $14 \mathrm{~kb}$ in length and contains 15 exons. The SLC11A1 protein is a $\mathrm{pH}$-dependent proton/divalent cation antiporter involved in macrophage function. The Slc11a1 gene in mice has been implicated in susceptibility to inflamma- tory autoimmune disorders [28] and to certain intracellular pathogens such as Mycobacteria, Leishmania and Salmonella [29]. Although the mechanism by which SLC11A1 controls intracellular pathogens remains unclear, polymorphisms of human SLC11A1 are associated with susceptibility to several inflammatory autoimmune disorders as well as infectious diseases including leprosy, TB, visceral leishmania (VL) and human immunodeficiency virus (HIV). Two independent studies in the Gambian population confirm the association between SLC11A1 and susceptibility to TB infection [30,31] (table 6). However, other association studies were unable to link SLC11A1 with susceptibility to TB in Moroccans [32].

Furthermore, a study of linkage disequilibrium (LD) of 8 SNPs across the $14 \mathrm{~kb}$ SLC11A1 gene provides evidence that two distinct patterns of tight LD exist for this gene in a Chinese population; there is high LD among markers in the 5'region and among markers in the 3'region, but not between these regions [34]. A similar pattern was observed between markers in the 5' and 3 'region of the gene in a Gambian population.

Therefore, it is critical to identify and use markers from across the entire gene for use in association and linkage studies of disease susceptibility. Microsatellites previously identified at this locus include a Z-DNA forming (GT)n dinucleotide repeat in the promoter region of the SLC11A1 gene reported to have enhancer activity and a tetranucleotide (CAAA)n repeat at the 3'UTR terminal region of the gene [35].

\section{Ras-Associated small GTP-ases}

The contribution of CD8+ T cells to the antimycobacterial host response includes:

a) cytotoxicity mediated via a granule-dependent exocytosis pathway;

b) cytotoxicity mediated via Fas/Fas ligand interaction;

c) direct microbicidal activity; and

d) release of cytokines [36].

Among relevant cytokines, $\mathrm{T}$ cell-derived interferon (IFN)-g is central to the protective host immune response. IFN-g is expressed on antigenspecific activation and leads to maturation of effector $\mathrm{T}$ cell functions and activation of infected macrophages. Macrophages play a dual role in the antimycobacterial host response: on the one hand, they restrict the growth of engulfed mycobacteria; on the other hand, they shield the pathogen from

Table 6. - SLC11A1 polymorphisms and their genotypes

\begin{tabular}{lll}
\hline Polymorphism & Genotype & Association with TB \\
\hline INT4 & CC & Significant in pulmonary TB [33] \\
\hline D543N & GA & Significant in pulmonary TB \\
& GG+ haplotype & Reduced frequency in patients \\
& CAdel & Reduced frequency in patients \\
\hline
\end{tabular}


being extinguished [37]. M. tuberculosis resides in phagosomal vesicles, where complex interactions between pathogen and host have evolved. A crucial element of mycobacterial survival involves the blockade of phagosomal maturation, which normally leads to the death of the engulfed microbes. In this process, the recruitment of regulatory enzymes to the phagosomal membrane is disrupted by M. tuberculosis.

Ras-associated small GTPase (Rab) 5 and Rab7 are essential mediators of these fusion events; Rab5 accumulates in the membranes of $\mathrm{M}$. tuberculosis-containing phagosomes, whereas recruitment of Rab7 is inhibited [38]. Rab molecules play decisive roles in the regulation of intracellular trafficking in various cell types and tissues [39]. In $\mathrm{T}$ cells, for example, Rab27A controls the exocytosis of lysosomes as an important step in the release of cytotoxic granules as part of antimicrobial $\mathrm{T}$ cell effector functions.

More than 60 Rab family members presumably exist in humans, and some of them play vital roles in distinct diseases; nonetheless, the function of the majority of them remains unknown. The Rab members that outstand are provided in the illustration together with the role they play in the infection. Broader implications for the crucial role played by regulatory GTPases against TB have arisen from mouse studies, in which LRG-47, a member of the IFN-g-inducible p47 GTPase family, has been shown to be essential for protection against TB [40] (table 7).

\section{Cytokine Polymorphisms connected with TB}

In TB infection plays an important role in the interaction between the pathogen and the host immune system. Thus, cytokines play an important role in anti-TB immune response. Among them, IFN-g and tumour necrosis factor-a (TNF-a) are associated with macrophage activation and en- hanced antimycobacterial capabilities in marine and human models.

The role of IFN-g as the main macrophage activating cytokine during infection with Mtb is well established in different experimental models. In humans, in vitro human macrophage anti-Mtb activity requires IFN-g. Lymphocytes from TB patients have diminished IFN-g production in response to mycobacterial antigens [45]. However, the best evidence for a role of IFN-g in resistance to mycobacterial infections has been obtained in families with mutations of the a and $b$ chains of the IFN-g receptor, the interleukin-12 (IL-12) p40 chain, and the IL-12Rb1 genes [46]. In these families, affected individuals have a mendelian susceptibility to mycobacterial disease, particularly to non-tuberculous mycobacteria.

TNF-a plays multiple roles in defense and pathologic responses in TB. In TNF-a gene-targeted mice, increased susceptibility and structural alterations in granuloma formation have been demonstrated. TNF-a is also considered a major factor in host-mediated damage of lung tissue [47]. However, the strongest evidence for a protective role of TNF-a in human TB is the high incidence of TB reactivation in patients with rheumatoid arthritis treated with monoclonal antibodies against TNF-a.

In contrast, interleukin-10 (IL-10) and transforming growth factor-b1 (TGF-b1) are considered macrophage-deactivating cytokines. TGF-b1 enhances intracellular growth of Mtb in human monocytes and downregulates IFN-g production. Monocytes from TB patients have been reported to exhibit enhanced production of TGFb1. Furthermore, TGF-b is known to promote collagen deposition and development of fibrotic lesions. IL-10deficient mice have increased antimycobacterial immunity. IL-10 downregulates TH1-induced response to Mtb, reactivates chronic pulmonary $\mathrm{TB}$ in mice, and reduces Mtb-induced apoptosis of murine and human macrophages [48].

Table 7. - Ras-associated small GTP-ase (Rab) polymorphisms and their functions

\begin{tabular}{|c|c|c|c|}
\hline Rab GTP-ase & Function & Locus & Association with TB \\
\hline Rab 5 & $\begin{array}{l}\text { Regulator of phagosome maturation, a process } \\
\text { that is targeted by } M \text {. tuberculosis }\end{array}$ & $7 q 11.21$ & \\
\hline Rab 7 & Regulator of phagosome maturation & $3 q 22.1$ & Slightly increased in patients \\
\hline Rab33 & $\begin{array}{l}\mathrm{CD} 8+\mathrm{T} \text { cell factor [41]. Killing of } M . \text { tuberculosis in } \\
\text { macrophages by CD8+ T cells via a perforin/granulysin- } \\
\text { mediated process has been described [42] }\end{array}$ & $\mathrm{Xq} 26$ & $\begin{array}{l}\text { Reduced gene expression } \\
\text { in patients with } \mathrm{TB}\end{array}$ \\
\hline Rab 24 & $\begin{array}{l}\text { Participate in fusion events between the endoplasmatic } \\
\text { reticulum/cis-Golgi compartment and lysosomes } \\
\text { that control autophagy processes[43]. Elimination } \\
\text { of M. tuberculosis within macrophages }\end{array}$ & $5 q 35.3$ & Increased in patients with $\mathrm{TB}$ \\
\hline Rab 13 & $\begin{array}{l}\text { Regulates continuous endocytic recycling in epithelial } \\
\text { cell lines [44] }\end{array}$ & $1 \mathrm{q} 21.2$ & Increased in patients with $\mathrm{TB}$ \\
\hline Rab 27A & Related to cytotoxic CD8+ T cell functions & $15 q 15-q 21.1$ & Slightly increased in patients with $\mathrm{TB}$ \\
\hline
\end{tabular}


Patients with TB have increased IL-10 production, mainly in anergic patients. However it is suggested that these findings show that IL-6 is involved in stimulating early IFN-g production, but is not essential for development of protective immunity against Mtb [49].

Studies on TB patients with different ethnic backgrounds have shown conflicting evidence for TNF-a and IL-10 -1082 polymorphisms association with TB. No association was found for TGFb. In contrast, published reports agree that IFNg+874 allele A, associated with low IFN-g production, is associated with pulmonary TB [50], nevertheless these reports do not differentiate between different clinical forms of TB (table 8).

\section{Mycobacterial antigens that play a role in latent or active TB}

It is necessary to make a short reference regarding the role of the mycobacterium itself in the establishment of the infection, so as to have a complete idea of the subject.

The abundance of regulatory proteins in the $\mathrm{M}$. tuberculosis genome may explain the ability of the pathogen to adapt to this hostile environment [51] by up-regulating so-called latency genes. One of the most prominent of these is the one that encodes Rv2031c (also known as a-crystallin, HspX, or the $16-\mathrm{kDa}$ antigen), whose importance is demonstrated by the reduced ability of bacteria deficient in this gene to grow in macrophages. Moreover, production of Rv2031c appears to increase as the bacteria go into the metabolically resting stage and to decrease as they revert to exponential growth [52]. It therefore serves as the prototypic "latency-associated antigen".

Moreover, although $\mathrm{M}$. tuberculosis is an obligatory aerobic organism, it is able to adapt to and survive in the hypoxic and hostile environment of host macrophages. In this regard, it has been shown that $\mathrm{M}$. tuberculosis undergoes a dramatic change in gene transcription characteristic of non-replicating persistence. When grown in vitro in oxygen-depleted cultures, as well as in cultures exposed to nitric oxide, M. tuberculosis up-regulates overlapping, characteristic sets of genes [53], among which is the gene encoding the small 16$\mathrm{kDa}$ heat shock protein Rv2031c. Expression of this protein is increased by a factor of approximately 4 to 7 during the stationary growth phase and appears to be crucial for survival of the organism [54].

In addition, $\mathrm{M}$. tuberculosis strains of the $\mathrm{W}$ Beijing genotype acquired mis-sense mutations in DNA repair genes [55]. These M. tuberculosis WBeijing genotype strains are genetically highly conserved and widespread.

The success of this group of strains may result in part from mutations in DNA repair enzymes, which might provided a true selective advantage for these bacteria to adapt and persist, including through the acquisition of resistance to anti-TB drugs. Mutations in the DNA repair genes might be the evolutionary answer of the TB bacillus to increase adaptation to hosts. This adaptation will lead to increasing trends in the TB epidemic in the coming decades.

\section{Conclusions}

In conclusion, we have to assume that more research and development is needed to determine the efficacy of new tests in detecting latent TB in both developed and developing countries [56].

Moreover, the role of human genes in the beginning and progression of pulmonary tuberculosis has to be clarified. The use of proteomics and transcriptomic analyses will help in the identification of new genes and we hope that using simple genetic tests will assist as a guide in attempting to control and cure tuberculosis.

Table 8. - Cytokine polymorphisms involved in TB infections

\begin{tabular}{lll}
\hline Cytokine & Polymorphism & Consequnces/associations \\
\hline IL-10 & $-1082(\mathrm{~A} / \mathrm{A})$ & Low IL-10 production in patients with pleural TB \\
\hline IFN-g & $+874(\mathrm{~A}$ allele) & Higher frequency in controls and pulmonary TB patients \\
\cline { 2 - 3 } & $+874(\mathrm{~T} / \mathrm{T})$ & Decreased in pulmonary TB, but not in pleural or miliary \\
\hline TNF-a & -308 & \\
\hline TGF-b1 & $-238(\mathrm{G} / \mathrm{A})$ & \\
\cline { 2 - 3 } & $+509 \mathrm{C}-\mathrm{T}$ & In linkage disequilibrium with -509 C-T \\
\hline IL-6 & +915 G-C & Similar distribution in healthy and patients to +29 T-C \\
\hline
\end{tabular}




\section{References}

1. Krutzik SR, Modlin RL. The role of Toll-like receptors in combating mycobacteria. Semin Immunol 2004; 16: 35-41.

2. Padmini Salgame. Host innate and Th1 responses and the bacterial factors that control Mycobacterium tuberculosis infection. Current Opinion in Immunology 2005; 17: 374-380.

3. Hill AV. The genomics and genetics of human infectious disease susceptibility. Аппи Rev Genom Hum Genet 2001 2: 373-400.

4. North RJ, Jung YJ. Immunity to tuberculosis. Annu Rev Immunol 2004; 22: 599-623.

5. Ogata K, Linzer BA, Zuberi RI, et al. A. Activity of defensins from human neutrophilic granulocytes against Mycobacterium avium-Mycobacterium intracellulare. Infect Immun 1992; 60: 4720-5.

6. Ratcliffe LT, Lukey PT, MacKenzie CR, et al. Reduced NK activity correlates with active disease in HIVpatients with multidrug-resistant pulmonary tuberculosis. Clin Exp Immunol 1994; 97: 373-9.

7. Denis M. Killing of Mycobacterium tuberculosis within human monocytes: activation by cytokines and calcitriol. Clin Exp Immunol 1991; 84: 200-6.

8. Saltini C. Chemotherapy and diagnosis of tuberculosis. Resp Med 2006; 100: 2085-2097.

9. Meyer CG, May J, Starkx K. Human leukocyte antigens in tuberculosis and leprosy. Trends Microbiol 1998; 6: 148-154.

10. Goldfeld AE, Delgado JC, et al. Association of an HLA-DQ allele with clinical tuberculosis. JAMA 1998; 279: 226-228.

11. Dubaniewicz A, Moszkowska G, Szczerkowska, et al. Analysis of DQB1 allele frequencies in pulmonary tuberculosis: preliminary report. Thorax 2003; 58, 890-891.

12. Hill RJ, Zozulya S, Lu YL, et al. The lymphoid protein tyrosine phosphatase Lyp interacts with the adaptor molecule Grb2 and functions as a negative regulator of T-cell activation. Exp Hematol 2002; 30: 237-44.

13. Cloutier JF, Veillette A. Cooperative inhibition of Tcell antigen receptor signaling by a complex between a kinase and a phosphatase. J Exp Med 1999; 189: 111.

14. Gomez LM, Anaya J-M, Martin J. Genetic Influence of PTPN22 R620W Polymorphism in Tuberculosis. Human Immunology 2005; 66: 1242-1247.

15. Means TK, Jones BW, Schromm AB, et al. Differential effects of a Toll-like receptor antagonist on Mycobacterium tuberculosis-induced macrophage responses. $J$ Immunol 2001; 166: 4074-4082.

16. Supajatura V, Ushio H, Nakao A, et al. Differential responses of mast cell Toll-like receptors 2 and 4 in allergy and innate immunity. J Clin Invest 2002; 109: 1351-1359.

17. Means TK, Wang S, Lien E, Yoshimura A, et al. Human toll-like receptors mediate cellular activation by Mycobacterium tuberculosis. J Immunol 1999; 163: 3920-3927.

18. Thoma-Uszynski S, Stenger S, Takeuchi O, et al. Induction of direct antimicrobial activity through mammalian Toll-like receptors. Science 2001; 291: 1544-1547.

19. Lorenz E, Mira JP, Cornish KL, et al. A novel polymorphism in the Toll-like receptor 2 gene and its potential association with staphylococcal infection. Infect Immun 2000; 68: 6398-6401.

20. Ogus AC, Yoldas B, Ozdemir T, et al. The Arg753Gln polymorphism of the human Toll-like receptor 2 gene in tuberculosis disease. Eur Respir J 2004; 23: 219-223.

21. Liu PT, Stenger S, Li H, et al. Toll-Like Receptor triggering of a Vitamin D-Mediated Human Antimicrobial response. Science 2006; 311: 1770-1773.

22. Bellamy R. Susceptibility to mycobacterial infections: The importance of host genetics. Genes Immun 2003; 4: $4-11$.
23. Tsoukas CD, Provvedini DM, Manolagas SC. 1,25-dihydroxy vitamin D3: A novel immunoregulatory hormone. Science 1984; 224: 1438-1440.

24. Malloy PJ, Feldman D. Vitamin D resistance. Am J Med 1999; 106: 355-370.

25. Arai H, Miyamoto K, Taketani Y, et al. A vitamin D receptor gene polymorphism in the translation initiation codon: Effect on protein activity and relation to bone mineral density in Japanese women. J Bone Miner Res 1997; 12: 915-921.

26. Sharma OP. Hypercalcemia in granulomatous disorders. A clinical review. Curr Opin Pul Med 2000; 6: 442-447.

27. Govoni G, Vidal S, Cellier M, et al. Genomic structure, promoter sequence, and induction of expression of the mouse Nramp1 gene in macrophages. Genomics 1995; 27: 9.

28. Wicker LS, Chamberlain G, Hunter, et al. Fine mapping, gene content, comparative sequencing, and expression analyses support Ctla4 and Nramp1 as candidates for Idd5.1 and Idd5.2 in the nonobese diabetic mouse. J Immunol 2004; 73: 164-73.

29. Vidal S, Tremblay ML, et al. The Ity/Lsh/Bcg locus: natural resistance to infection with intracellular parasites is abrogated by disruption of the Nramp1 gene. $J$ Exp Med 1995; 182: 655.

30. Bellamy R, Beyers N, McAdam K, et al. Genetic susceptibility to tuberculosis in Africans: a genome-wide scan. PNAS 2000; 97: 8005.

31. Awomoyi AA, Marchant, et al. Interleukin-10, polymorphism in SLC11A1 (formerly NRAMP1), and susceptibility to tuberculosis. J Infect Dis 2002; 186: 1808.

32. El Baghdadi J, Remus N, Benslimane. Variants of the human NRAMP1 gene and susceptibility to tuberculosis in Morocco. Intern J Tubercle Lung Dis 2003; 7: 599.

33. Taype CA, Castro JC, Accinelli RA, et al. Espinoza. Association between SLC11A1 polymorphisms and susceptibility to different clinical forms of tuberculosis in the Peruvian population. Infection, Genetics and Evolution 2006; 6: 361-367.

34. Yip SP, Leung KH, Lin CK. Extent and distribution of linkage disequilibrium around the SLC11A1 locus. Genes and Immunity 2003; 4: 212.

35. Buu NT, Cellier M, Gros P, et al. Identification of a highly polymorphic length variant in the 3'UTR of NRAMP1. Immunogenetics 1995; 42: 428.

36. Lazarevic V, Flynn J. CD8+ T cells in tuberculosis. Am $J$ Respir Crit Care Med 2002; 166: 1116-21.

37. Kaufmann SH. How can immunology contribute to the control of tuberculosis? Nat Rev Immunol 2001; 1: 20-30.

38. Via LE, Deretic D, Ulmer RJ, et al. Arrest of mycobacterial phagosome maturation is caused by a block in vesicle fusion between stages controlled by rab5 and rab7. J Biol Chem 1997; 272: 13326-31.

39. Zerial M, McBride H. Rab proteins as membrane organizers. Nat Rev Mol Cell Biol 2001; 2: 107-17.

40. MacMicking JD, Taylor GA, McKinney JD. Immune control of tuberculosis by IFN-gamma-inducible LRG47. Science 2003; 302: 654-9.

41. Bellamy R, Beyers N, McAdam KP, et al. Genetic susceptibility to tuberculosis in Africans: a genome-wide scan. Proc Natl Acad Sci USA 2000; 97: 8005-9.

42. Stenger S, Hanson DA, Teitelbaum R, et al. An antimicrobial activity of cytolytic $\mathrm{T}$ cells mediated by granulysin. Science 1998; 282: 121-5.

43. Gutierrez MG, Master SS, Singh SB, et al. Autophagy is a defense mechanism inhibiting BCG and Mycobacterium tuberculosis survival in infected macrophages. Cell 2004; 119: 753-66.

44. Morimoto S, Nishimura N, Terai T, et al. Rab13 mediates the continuous endocytic recycling of occludin to the cell surface. J Biol Chem 2004; 280: 2220-8. 
45. Sanchez FO, Rodriguez JI, Agudelo G, et al. Immune responsiveness and lymphokine production in patients with tuberculosis and healthy controls. Infect Immun 1994; 62: 5673-8.

46. Altare F, Jouanguy E, Lamhamedi S, et al. Mendelian susceptibility to mycobacterial infection in man. Curr Opin Immunol 1998; 10: 413-7.

47. Bean AGD, Roach DR, Briscoe H, et al. Structural deficiencies in granuloma formation in TNF gene-targeted mice underlie the heightened susceptibility to aerosol Mycobacterium tuberculosis infection, which is not compensated for by lymphotoxin. J Immunol 1999; 162: 3504-11.

48. Gil DP, Leon LG, Correa LI, et al. Differential induction of apoptosis and necrosis in monocytes from patients with tuberculosis and healthy controls. J Infect Dis 2004; 189: 2120-8.

49. Saunders BM, Frank AA, Orme IM, et al. Interleukin-6 induces early gamma interferon production in the infected lung but is not required for generation of specific immunity to Mycobacterium tuberculosis infection. Infect Immun 2000; 68: 3322-6.

50. Rossouw M, Nel HJ, Cooke GS, et al. Association between tuberculosis and a polymorphic NFkappaB bind- ing site in the interferon gamma gene. Lancet 2003; 361: 1871-2.

51. Honer zu Bentrup K, Russell DG. Mycobacterial persistence: adaptation to a changing environment. Trends Microbiol 2001; 9: 597-605.

52. Hu Y, Coates AR. Transcription of the stationaryphase-associated hspX gene of Mycobacterium tuberculosis is inversely related to synthesis of the 16-kilodalton protein. J Bacteriol 1999; 181: 1380-1387.

53. Voskuil MI, Schnappinger D, Visconti KC, et al. Inhibition of respiration by nitric oxide induces a Mycobacterium tuberculosis dormancy program. $J$ Exp Med 2003; 198: 705-713.

54. Yuan Y, Crane DD, Simpson RM, et al. The 16-kDa acrystallin (Acr) protein of Mycobacterium tuberculosis is required for growth in macrophages. Proc Natl Acad Sci USA 1998; 95: 9578-9583.

55. Ebrahimi Rad M, Bifani P, Martin C, et al. Mutations in putative mutator genes of Mycobacterium tuberculosis strains of the W-Beijing family. Emerg Infect Dis 2004; 10: $1337-8$.

56. Heinke Kunst. Diagnosis of latent tuberculosis infection: The potential role of new technologies. Resp Med 2006; 100, 2098-2106.

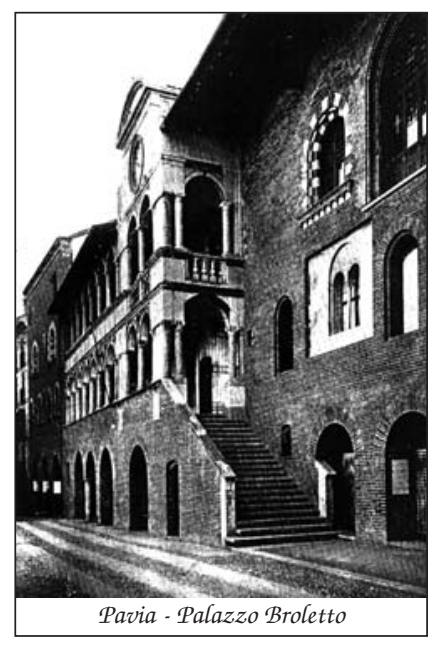

